\title{
Valence-Band Structure of the Ferromagnetic Semiconductor GaMnAs Studied by Spin-Dependent Resonant Tunneling Spectroscopy
}

\author{
Shinobu Ohya ${ }^{1,2}$ Iriya Muneta, ${ }^{1}$ Pham Nam Hai, ${ }^{1}$ and Masaaki Tanaka ${ }^{1}$ \\ ${ }^{1}$ Department of Electrical Engineering and Information Systems, The University of Tokyo, \\ 7-3-1 Hongo, Bunkyo-ku, Tokyo 113-8656, Japan \\ ${ }^{2}$ Japan Science and Technology Agency, 4-1-8 Honcho, Kawaguchi, Saitama 332-0012, Japan \\ (Received 20 October 2009; revised manuscript received 12 April 2010; published 22 April 2010)
}

\begin{abstract}
The valence-band structure and the Fermi level $\left(E_{\mathrm{F}}\right)$ position of ferromagnetic-semiconductor GaMnAs are quantitatively investigated by electrically detecting the resonant tunneling levels of a GaMnAs quantum well $(\mathrm{QW})$ in double-barrier heterostructures. The resonant level from the heavy-hole first state is clearly observed in the metallic GaMnAs $\mathrm{QW}$, indicating that holes have a high coherency and that $E_{\mathrm{F}}$ exists in the band gap. Clear enhancement of tunnel magnetoresistance induced by resonant tunneling is demonstrated in these double-barrier heterostructures.
\end{abstract}

GaMnAs is a prototype ferromagnetic semiconductor $[1,2]$, in which many unique phenomena induced by the combination of its magnetic and semiconducting properties have been observed. In the last decade, a variety of its physical properties have been investigated from the physics point of view as well as the application point of view for future spintronic devices. In spite of these extensive studies, however, a complete understanding of the electronic structure of GaMnAs has not been achieved yet. As the mechanism of ferromagnetism in GaMnAs, the mean-field Zener model $[3,4]$, where the ferromagnetism is induced by the interaction between the valence-band (VB) holes up to $10^{20}-10^{21} \mathrm{~cm}^{-3}$ and the localized $\mathrm{Mn}-3 d$ electrons, explained a variety of phenomena observed in GaMnAs. In this model, it is assumed that the Fermi level $\left(E_{\mathrm{F}}\right)$ position is determined by the concentration of the VB holes and that $E_{\mathrm{F}}$ lies in VB at 200-300 meV lower than the VB top. On the other hand, several recent reports on the optical [5-7] and transport [8,9] properties of GaMnAs have shown that $E_{\mathrm{F}}$ exists in the impurity band (IB) within the band gap of GaMnAs [10], generating dispute on the band structure and the mechanism of the ferromagnetism in GaMnAs [11].

Resonant tunneling spectroscopy is a very powerful method for investigating the band structure because the resonant levels contain a lot of information, such as the effective mass, band offset, and $E_{\mathrm{F}}$. Previously, we successfully observed the resonant tunneling effect in doublebarrier resonant tunneling diodes (RTDs) containing a GaMnAs quantum well (QW) with the thicknesses from 3.8 to $20 \mathrm{~nm}$ at $2.6 \mathrm{~K}$ [12]. Our results indicated that the mean free path of the VB holes is longer than $20 \mathrm{~nm}$ in GaMnAs. However, the Curie temperature $\left(T_{\mathrm{C}}\right)$ of the GaMnAs QW was limited to less than $\sim 30 \mathrm{~K}$ because the interstitial $\mathrm{Mn}$ atoms cannot be easily removed out of the GaMnAs QW during the growth [13,14]. In such a case, GaMnAs has a relatively small hole concentration and shows insulating behavior which is quite different from that of metallic GaMnAs with higher $T_{\mathrm{C}}$.

Recently, we found that $T_{\mathrm{C}}$ of the inside GaMnAs layer can be increased to $60-70 \mathrm{~K}$ even when its thickness is thin $(2 \mathrm{~nm})$ by using a paramagnetic AlMnAs tunnel barrier in the GaMnAs single-barrier heterostructures [15]. This improvement is probably because the interstitial $\mathrm{Mn}$ atoms can more easily pass through the AlMnAs barrier than through the Mn-free barrier during the growth. In this Letter, we investigate the resonant levels formed in the metallic GaMnAs QW [16] with a $T_{\mathrm{C}}$ value of $60 \mathrm{~K}$, and show that the high coherency of the VB holes is a typical feature of GaMnAs and that $E_{\mathrm{F}}$ is not in VB but in the band gap. Using the observed resonant levels, we quantitatively analyze the $\mathrm{VB}$ structure and the $E_{\mathrm{F}}$ position of the GaMnAs QW.

Figure 1(a) shows the schematic cross sectional RTD structures grown by molecular beam epitaxy comprising, from the surface side, $\mathrm{Ga}_{0.94} \mathrm{Mn}_{0.06} \mathrm{As}(10 \mathrm{~nm}) /$ $\operatorname{GaAs}(1 \mathrm{~nm}) /\left(\mathrm{Al}_{0.5} \mathrm{Ga}_{0.5} \mathrm{As}\right.$ or $\left.\mathrm{Al}_{0.94} \mathrm{Mn}_{0.06} \mathrm{As}\right)(4 \mathrm{~nm}) /$ $\operatorname{GaAs}(2 \mathrm{~nm}) / \mathrm{Ga}_{0.94} \mathrm{Mn}_{0.06} \operatorname{As}(2.5 \mathrm{~nm}) / \operatorname{GaAs}(1 \mathrm{~nm}) /$ $\operatorname{AlAs}(4 \mathrm{~nm}) /$ Be-doped $\operatorname{GaAs}(100 \mathrm{~nm})$ on a $p$-type GaAs(001) substrate. We prepared two RTD samples named as RTD $A$ and $B$ with the upper tunnel barrier composed of $\mathrm{Al}_{0.5} \mathrm{Ga}_{0.5} \mathrm{As}$ and $\mathrm{Al}_{0.94} \mathrm{Mn}_{0.06} \mathrm{As}$, respectively. The $\mathrm{Be}$ concentration of the Be-doped GaAs (GaAs:Be) layer was $2 \times 10^{18} \mathrm{~cm}^{-3}$. The thin GaAs spacer layers were inserted to smooth the surface. The $\mathrm{GaAs}: \mathrm{Be}, \mathrm{AlAs}$, and the lowest GaAs spacer layers were grown at high temperatures of 600,550 , and $600^{\circ} \mathrm{C}$, respectively. The GaMnAs QW, GaAs/(AlGaAs or AlMnAs)/GaAs, and the top GaMnAs layers were grown at low temperatures of 240,200 , and $225^{\circ} \mathrm{C}$, respectively. We confirmed that the AlMnAs layer is paramagnetic even at $3 \mathrm{~K}$ by the magnetic-field dependence of the magnetic circular dichroism measurements. After the growth, circular mesa diodes with $200 \mu \mathrm{m}$ in diameter were fabricated 

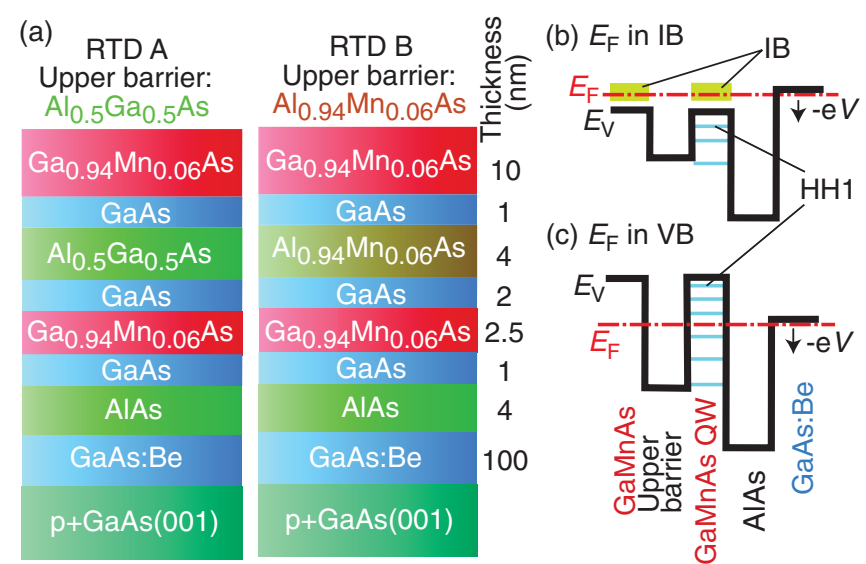

FIG. 1 (color). (a) Schematic cross sectional structures of RTD $A$ and $B$. (b) (c) The schematic band diagrams of our RTD structures assuming that $E_{\mathrm{F}}$ exists in IB and $\mathrm{VB}$ in GaMnAs, respectively. The black and blue lines are VB at the $\Gamma$ point $\left(E_{V}\right)$ and quantum levels of $\mathrm{VB}$, respectively. The red dash-dotted lines are $E_{\mathrm{F}}$. The green bands are IB in (b).

by chemical etching. We spin coated an insulating negative resist on the sample, opened a contact hole with $180 \mu \mathrm{m}$ in diameter on the top of the mesa, and fabricated a metal electrode by evaporating $\mathrm{Au}$ on this surface. In our transport measurements, the bias polarity is defined by the voltage of the top GaMnAs electrode with respect to the substrate.

Figures 1(b) and 1(c) show the schematic band diagrams of our RTD structures when assuming that $E_{\mathrm{F}}$ exists in IB and VB in the GaMnAs QW, respectively. The black and blue lines are $\mathrm{VB}$ at the $\Gamma$ point $\left(E_{\mathrm{V}}\right)$ and quantum levels of $\mathrm{VB}$, respectively. The red dash-dotted lines are $E_{\mathrm{F}}$. Since the resonant levels are detected when holes are injected from GaAs:Be in our RTD devices [12], only the resonant levels below $E_{\mathrm{F}}$ can be detected. Therefore, the heavy-hole first state (HH1) can be seen in the case of Fig. 1(b), not Fig. 1(c). In our devices, the parallel and antiparallel magnetization alignments can be switched by sweeping the magnetic field along the [100] axis in plane because of the difference of the coercivities between the GaMnAs layers [12]. The $d I / d V-V, d^{2} I / d V^{2}-V$, and TMR- $V$ data were numerically derived from the $I-V$ data at zero magnetic field.

Figures 2(a) and 2(b) show the $d I / d V-V$ and $d^{2} I / d V^{2}-V$ characteristics of RTD devices fabricated in this study, respectively. In each graph, the blue and red (green and orange) curves are the data of RTD $A(B)$. The blue or green curves correspond to the parallel magnetization $(\mathrm{P})$. The red or orange curves correspond to the antiparallel magnetization (AP). In Fig. 2(a), oscillations induced by resonant tunneling are observed in the negative bias region in both RTD devices. These resonant peaks are more clearly seen in Fig. 2(b). The shapes of the $d^{2} I / d V^{2}-V$ curves of both RTD $A$ and $B$ are quite similar to those observed in other RTD devices with the various GaMnAs
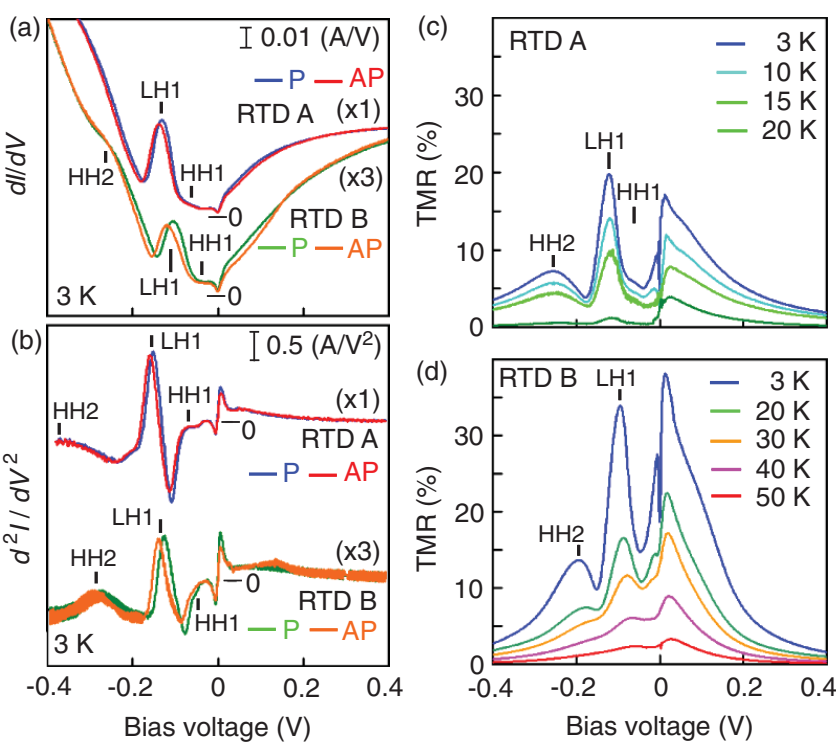

FIG. 2 (color). (a) $d I / d V-V$ and (b) $d^{2} I / d V^{2}-V$ characteristics of RTD $A$ and $B$ at $3 \mathrm{~K}$. In both graphs, the blue and red (green and orange) curves correspond to RTD $A(B)$. The blue or green curve corresponds to the parallel magnetization $(\mathrm{P})$. The red or orange curve corresponds to the antiparallel magnetization (AP). (c) and (d) show the temperature evolution of the bias dependence of TMR obtained in RTD $A$ and $B$, respectively.

QW thicknesses in our previous study [12]. Thus, these resonant peaks can be assigned as described in Figs. 2(a) and 2(b). Here, $\mathrm{HH} n$ and $\mathrm{LH} n(n=1,2,3, \ldots)$ are resonant tunneling through the $n$th level of the heavy-hole (HH) band and light-hole (LH) band in the GaMnAs QW, respectively. The resonant peaks in RTD $A$ are observed at higher voltages than those in RTD $B$, which is due to the barrier height difference between $\mathrm{Al}_{0.5} \mathrm{Ga}_{0.5} \mathrm{As}$ $(\sim 225 \mathrm{meV})$ and $\mathrm{Al}_{0.94} \mathrm{Mn}_{0.06} \mathrm{As}(\sim 110 \mathrm{meV})$ [15]. The peak positions are different between $\mathrm{P}$ and AP only at the LH1 state both in RTD $A$ and $B$, which is consistent with the characteristics of the $p-d$ exchange interaction with the in-plane magnetization of GaMnAs [12,17]. The difference in the resonant peaks' voltage at LH1 between $\mathrm{P}$ and AP is 8 and $13 \mathrm{mV}$ in RTD $A$ and $B$, respectively. These values are related to the difference of the $T_{\mathrm{C}}$ values of the GaMnAs QW: $30 \mathrm{~K}$ (RTD $A$ ) and $60 \mathrm{~K}$ (RTD B). In Figs. 2(a) and 2(b), the resonant level of HH1 is observed both in RTD $A$ and $B$, which indicates that HH1 is not occupied by holes and $E_{\mathrm{F}}$ lies in the band gap as described in Fig. 1(b) in both RTDs.

Figures 2(c) and 2(d) show the temperature evolution of the bias dependence of the tunnel magnetoresistance (TMR) obtained in RTD $A$ and $B$, respectively. In both RTD devices, clear enhancement of TMR is observed especially at LH1 reflecting the spin splitting at LH1 as shown in (a) and (b). It is a quite unique property that the TMR ratio at LH1 is higher than that at zero bias in RTD A at 3-15 K. Such clear TMR enhancements induced by 
resonant tunneling are due to the high crystallinity of GaMnAs and have never been observed in the studies of magnetic tunnel junctions of other material systems $[14,18]$. TMR disappears at $30 \mathrm{~K}$ in RTD $A$ and at $60 \mathrm{~K}$ in RTD $B$ with increasing temperature, which indicates that the $T_{\mathrm{C}}$ of the GaMnAs QW is estimated to be $30 \mathrm{~K}$ and $60 \mathrm{~K}$ in RTD $A$ and $B$, respectively. We note that the top GaMnAs layer has a $T_{\mathrm{C}}$ of $60 \mathrm{~K}$ which was determined by magnetic circular dichroism.

We calculated the quantum levels in these heterostructures by the multiband transfer matrix technique developed in Ref. [19] with the Luttinger-Kohn $6 \times 6 k \cdot p$ Hamiltonian and $p-d$ exchange interaction [3]. In Fig. 3(a), the thin black curves, thick blue lines, and red dash-dotted line correspond to the $E_{\mathrm{V}}$ position estimated in our RTD structures, $E_{\mathrm{V}}$ assumed in our calculation, and $E_{\mathrm{F}}$, respectively. In the $E_{\mathrm{V}}$ diagrams shown in Fig. 3(a), the quantization, strain, and exchange splitting are neglected. We note that the $E_{\mathrm{V}}$ positions with respect to $E_{\mathrm{F}}$ are different between the top GaMnAs and the GaMnAs QW in this picture (see the black curves), which is due to the quantumsize effect explained later. In our calculation of the quantum levels, we neglected the small band-bending effect and assumed a flat band structure as expressed by the thick blue lines. Furthermore, we replaced the GaMnAs top electrode with the GaAs:Be electrode, because the character of the wave function of GaMnAs is unknown, and this replacement does not largely affect the calculated results of the quantum levels. Here, we define $\Delta_{x}$ and $E_{\text {Diff }}$ as the exchange splitting energy for the LH band in the in-plane [100] direction and the $E_{\mathrm{V}}$ position of the GaMnAs QW with respect to that of the GaAs:Be electrode, respectively. We neglected the small strain effect in the GaMnAs QW.

The values of $\Delta_{x}$ and $E_{\text {Diff }}$ were determined as follows. Figure 3(b) shows the experimentally obtained resonantpeak bias voltage $V_{\mathrm{R}}$ in the $d^{2} I / d V^{2}-V$ characteristics as a (a)

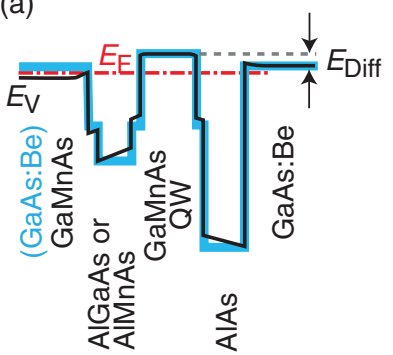

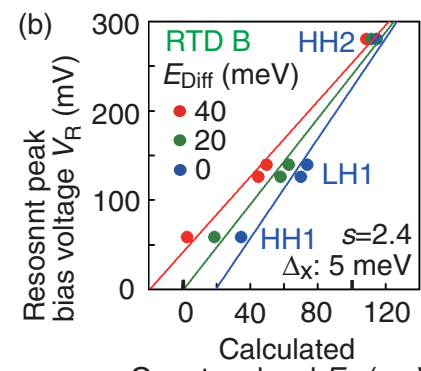

Quantum level $E_{\mathrm{R}}(\mathrm{meV})$
FIG. 3 (color). (a) Thin black curves, thick blue lines, and red dash-dotted line are the $E_{\mathrm{V}}$ positions estimated in our RTD structures, $E_{\mathrm{V}}$ assumed in our calculation of the quantum levels, and $E_{\mathrm{F}}$, respectively. Here, the quantization, strain, and exchange splitting are neglected. (b) Experimentally obtained resonantpeak bias voltage $V_{\mathrm{R}}$ as a function of the calculated resonant level $E_{\mathrm{R}}$ with respect to $E_{\mathrm{F}}$ in RTD $B$ with various $E_{\text {Diff }}$ when $\Delta_{x}$ is fixed at $5 \mathrm{meV}$. function of the calculated resonant level $E_{\mathrm{R}}$ with respect to $E_{\mathrm{F}}$ in RTD $B$ with various $E_{\text {Diff }}$ when $\Delta_{x}$ is fixed at $5 \mathrm{meV}$. Note that there are two points plotted at LH1 corresponding to the resonant peaks in P and AP. $E_{\text {Diff }}$ and $\Delta_{x}$ can be determined so that the relationship between $E_{\mathrm{R}}$ and $V_{\mathrm{R}}$ becomes linear and goes through the origin; $V_{\mathrm{R}}=s E_{\mathrm{R}}$ [20], where $s$ is the slope of the line. $\Delta_{x}, E_{\text {Diff }}$, and $s$ were estimated to be 3 (5) $\mathrm{meV}, 30$ (20) $\mathrm{meV}$, and 3.0 (2.4) for RTD $A(B)$, respectively. The $s$ values are a little larger than the ideal value of 2 (corresponding to the case that the equal bias drops occur at the two barriers in RTD), which is probably due to a little voltage drop at the interface between the top GaMnAs layer and the Au electrode. The $T_{\mathrm{C}}$ difference between RTD $A$ and $B$ can be seen as the $\Delta_{x}$ difference of 3 and $5 \mathrm{meV}$.

The black curves in Fig. 4(a) show the calculated VB structures of the GaAs:Be (left figure) and the GaMnAs QW when the AlGaAs (middle figure, RTD $A$ ) and AlMnAs (right figure, RTD $B$ ) are used as the top tunnel barrier, where the best fitting parameters obtained above are used and the quantization is not considered. The hori-
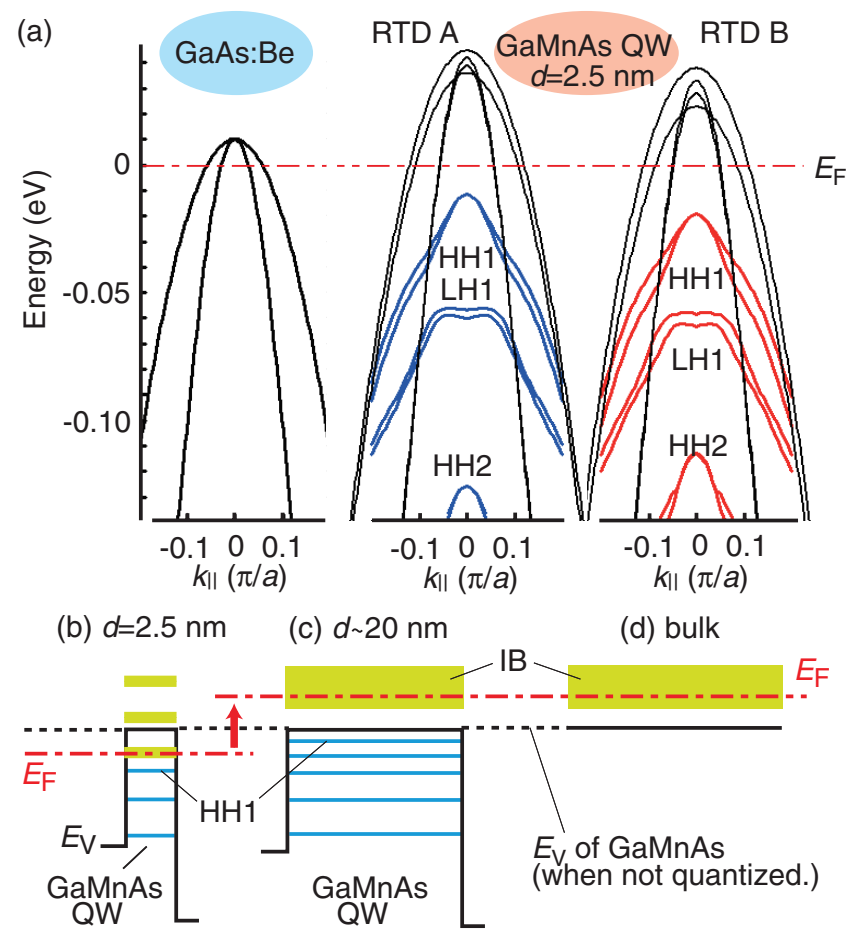

FIG. 4 (color). (a) Calculated VB structures (black curves) of the GaAs:Be and the GaMnAs QW with the calculated subbands (colored curves) when the AlGaAs (middle figure) and AlMnAs (right figure) are used as the top tunnel barrier, where the best fitting parameters obtained in this study are used. (b)(d) Schematic picture of the quantization of IB in the GaMnAs QW with the thickness $d$ of (b) $2.5 \mathrm{~nm}$, (c) $\sim 20 \mathrm{~nm}$, and (d) infinity (i.e., bulk). The black, blue, and green lines are $E_{\mathrm{V}}$, quantum levels of $\mathrm{VB}$, and the ones of the IB, respectively. The green bands are IB. Here, the exchange splitting and strain are neglected for simplicity. 
zontal axis is the in-plane wave number $k_{\|}$in the [100] direction. The calculated sub-bands formed by the quantization in the GaMnAs QW are also shown on these VB curves (red and blue curves). Note that the effective VB top corresponds to the top of the HH1 sub-band in each GaMnAs QW. From these results, we can see that $E_{\mathrm{F}}$ exists in the band gap (i.e., above the HH1 sub-band) of the GaMnAs QW in both RTD devices.

If we used the following parameters of GaMnAs assuming that $E_{\mathrm{F}}$ lies in the $\mathrm{VB}$, the carrier concentration of $4 \times$ $10^{20} \mathrm{~cm}^{-3}$, mobility $\mu$ of $2 \mathrm{~cm}^{2} / \mathrm{V} \mathrm{s}$ [9], and the Fermi wave vector $k_{F=} 0.4 \pi / a$ [3], the mean free path given by $\hbar k_{F} \mu / e$ ( $\hbar$ : Plank constant, $e$ : electron charge) would be less than $2 \mathrm{~nm}$. Thus, this model contradicts the high coherency of the VB holes obtained in our study. This consideration also supports our conclusions that $E_{\mathrm{F}}$ exists in the band gap and that the VB holes are not largely affected by the Mn impurities.

Here, we comment on the $E_{\text {Diff }}$ difference from our previous results in Ref. [12] where $E_{\text {Diff }}$ was estimated to be $\sim-30 \mathrm{meV}$ when the GaMnAs QW thickness was up to $20 \mathrm{~nm}$. Since $E_{\text {Diff }}$ obtained in this study is $\sim+20-30 \mathrm{meV}$, this result means that the increase in $E_{\text {Diff }}$ is $50-60 \mathrm{meV}$ by reducing the QW thickness from 20 to $2.5 \mathrm{~nm}$. A possible reason is the quantization of IB in the GaMnAs QW. This is schematically explained in Fig. 4 when the QW thickness $d$ is (b) $2.5 \mathrm{~nm}$, (c) $\sim 20 \mathrm{~nm}$, and (d) infinity (i.e., bulk). The black, blue, and green lines are $E_{\mathrm{V}}$, quantum levels of $\mathrm{VB}$, and the ones of IB, respectively. Here, the exchange splitting and strain are neglected. When the QW width is thin as shown in Fig. 4(b), $E_{\mathrm{F}}$ becomes lower than the original VB top of the GaMnAs due to the quantum-size effect of IB. When the QW becomes thick as shown in Fig. 4(c), quantization of IB becomes weaker. Thus, $E_{\mathrm{F}}$ is pushed up into the band gap so as to maintain the hole concentration. From these results, we can expect VB of the bulk GaMnAs as shown in Fig. 4(d).

In summary, we fabricated ferromagnetic-RTD devices with a GaMnAs QW whose $T_{\mathrm{C}}$ is 30 and $60 \mathrm{~K}$, and found that the HH1 state is not occupied by holes in both devices. This means that holes do not reside in VB of GaMnAs in the equilibrium condition; $E_{\mathrm{F}}$ exists in the band gap. The VB structure of GaMnAs can be reproduced by adding a small exchange splitting energy $\Delta_{x}$ of 3-5 meV (for the LH band) to the VB structure of GaAs. We clearly detected the enhancement of TMR induced by the resonant tunneling in these devices. Also, we found that the $E_{\mathrm{F}}$ shift of 50$60 \mathrm{meV}$ induced by the thickness reduction of the GaMnAs QW from 20 to $2.5 \mathrm{~nm}$, which is probably due to the quantization of IB.

This work was partly supported by Grant-in-Aids for Scientific Research, the Special Coordination Programs for Promoting Science and Technology, R\&D for Nextgeneration Information Technology by MEXT, Asahi Glass Foundation, and PRESTO of JST.

[1] H. Ohno, J. Magn. Magn. Mater. 200, 110 (1999); H. Ohno et al., Appl. Phys. Lett. 69, 363 (1996).

[2] M. Tanaka, J. Vac. Sci. Technol. B 16, 2267 (1998); T. Hayashi et al., J. Cryst. Growth 175-176, 1063 (1997).

[3] T. Dietl, H. Ohno, and F. Matsukura, Phys. Rev. B 63, 195205 (2001).

[4] T. Dietl et al., Science 287, 1019 (2000).

[5] V.F. Sapega et al., Phys. Rev. Lett. 94, 137401 (2005).

[6] K. S. Burch et al., Phys. Rev. Lett. 97, 087208 (2006).

[7] K. Ando et al., Phys. Rev. Lett. 100, 067204 (2008).

[8] L. P. Rokhinson et al., Phys. Rev. B 76, 161201(R) (2007).

[9] K. Alberi et al., Phys. Rev. B 78, 075201 (2008).

[10] J. Okabayashi et al., Phys. Rev. B 64, 125304 (2001).

[11] T. Jungwirth et al., Phys. Rev. B 76, 125206 (2007).

[12] S. Ohya, P. N. Hai, Y. Mizuno, and M. Tanaka, Phys. Rev. B 75, 155328 (2007).

[13] D. Chiba, K. Takamura, F. Matsukura, and H. Ohno, Appl. Phys. Lett. 82, 3020 (2003).

[14] E. Likovich et al., Phys. Rev. B 80, 201307(R) (2009).

[15] S. Ohya, I. Muneta, P. N. Hai, and M. Tanaka, Appl. Phys. Lett. 95, 242503 (2009).

[16] We confirmed the metallic feature of the GaMnAs QW in RTD $B$ by controlling the potential of QW in a three terminal device using RTD $B$ containing an electrode connected to QW. See S. Ohya, I. Muneta, and M. Tanaka, Appl. Phys. Lett. 96, 052505 (2010).

[17] M. Sawicki et al., Phys. Rev. B 70, 245325 (2004).

[18] T. Niizeki, N. Tezuka, and K. Inomata, Phys. Rev. Lett. 100, 047207 (2008).

[19] A. G. Petukhov, A. N. Chantis, and D. O. Demchenko, Phys. Rev. Lett. 89, 107205 (2002).

[20] M. Elsen et al., Phys. Rev. Lett. 99, 127203 (2007). 